\title{
Cytometry and Cytometers: Development and Growth
}

\author{
Howard M. Shapiro
}

\section{Overview}

It took almost 200 years of microscopy, from the mid-1600s until the mid-1800s, before objective data could be derived from specimens under the microscope by photography. The subsequent development of both image and flow cytometry for use by biologists followed the development of photometry, spectrometry, and fluorometry by physicists and chemists. Early cytometers measured cellular characteristics, such as nucleic acid content at the whole cell level; since few reagents were available that could specifically identify different types of cells, higher resolution imaging systems were developed for this task, but were too slow to be practical for many applications. The development of flow cytometry and cell sorting facilitated the development of more specific reagents, such as monoclonal antibodies and nucleic acid probes, which now allow cells to be precisely identified and characterized using simpler, low-resolution imaging systems. Although the most complex cytometers remain expensive, these newer instruments may bring the benefits of cytometry to a much wider community of users, including botanists in the field.

\section{1}

\section{Origins}

If the microscopic structures in cork to which Robert Hooke gave the name "cells" in the mid-17th century may be compared to the surviving stone walls of an ancient city, to what are we to compare the vistas available to 21st-century microscopists, who can follow the movements of individual molecules through living cells?

Between the time Hooke named them and the time that Schleiden, Schwann, and Virchow established cells as fundamental entities in plant and animal structure, function, and pathology, almost two centuries had elapsed. During most of that period, the only record of what could be seen under the microscope was an 
observer's drawing, and, even with the aid of a camera lucida, it was difficult if not impossible to eliminate subjective influences on the research product. The development of photography in the 1830 s was quickly followed by the marriage of the Daguerrotype camera and the microscope, but it was only in the $1880 \mathrm{~s}$ that photomicrography became accepted as the definitive objective method in microscopy, due in large measure to Robert Koch's advocacy (Breidbach 2002).

Even by that time, what we would today properly call cytometry, that is, the measurement of cells, was restricted to the quantification of morphologic characteristics, such as the sizes and numbers of cells and their organelles. The visualization of organelles themselves was greatly facilitated by differential staining methods, the development of which accelerated in the late 1800 s with the availability of newly synthesized aniline dyes (Baker 1958; Clark and Kasten 1983); Paul Ehrlich's initial researches in this area were to lead directly to the transformation of pharmacology from alchemy to science, and his appreciation of the specificity of antigen-antibody reactions provided an early milestone on the path toward modern immunochemical reagents.

Spectroscopy, a tool of physics adapted to chemistry and astronomy in the 19th century, became a mainstay of cytometry shortly thereafter. Microspectrophotometric measurement, either of intrinsic optical characteristics of cellular constituents or of optical properties of dyes or reagents added to cells, provided objective, quantitative information about cells' chemistry that could be correlated with their functional states.

The subsequent development of both cytometry and cytometers has been characterized by the use of such information, wherever possible in place of the inherently subjective and less quantitative results obtained by human observers.

In the remainder of this chapter, I will consider the history of cytometry from the 20th century onwards. Although much of the material has been covered, sometimes in greater detail, in several of my earlier publications (Shapiro 2003, 2004a, 2004b), this version of the story will pay special attention to one of the principal uses of cytometry in botany, namely, the determination of the genome sizes of plants by measurement of nuclear DNA content (Bennett and Leitch 2005; Doležel and Bartoš 2005; Greilhuber et al. 2005).

\section{2}

\section{From Absorption to Fluorescence, from Imaging to Flow}

It is easy, and probably easier for younger than for older readers, to forget that both Feulgen's staining procedure (Feulgen and Rossenbeck 1924) and Caspersson's ultraviolet (UV) absorption microspectrophotometric method for quantification of nuclear DNA content (Caspersson and Schultz 1938) were developed years before it was established that DNA was the genetic material. The evolution of cytometers from microscopes began in earnest in the 1930s in Torbjörn Caspersson's laboratory at the Karolinska Institute in Stockholm. He developed a series of progressively more sophisticated microspectrophotometers, and confirmed 
that, as had been suggested by conventional histologic staining techniques of light microscopy, tumor cells were likely to have abnormalities in DNA and RNA content (Caspersson 1950). In a memoir, which in itself provides useful insights on the development of cytometry, Leonard Ornstein (1987) documents the influence of Caspersson's work in establishing the genetic role of DNA. The first report that DNA contents of haploid, diploid, and tetraploid plant cells were in the ratio of 1:2:4 was published in 1950 by Swift, who made measurements using the Feulgen technique; his paper also introduced the terms $\mathrm{C}, 2 \mathrm{C}$, and $4 \mathrm{C}$ to describe the respective DNA contents for cells of a particular species.

\subsection{1}

\section{Early Microspectrophotometry and Image Cytometry}

Microspectrophotometers were first made by putting a small "pinhole" aperture, more properly called a field stop, in the image plane of a microscope, restricting the field of view to the area of a single cell, and placing a photodetector behind the field stop. Using progressively smaller field stops permits measurement of light transmission through correspondingly smaller areas of the specimen, and, by moving the stage in precise incremental steps in the plane of the slide, and recording the information, it becomes possible to measure the integrated absorption of a cell, and/or to make an image of the cell with each pixel corresponding in intensity to the transmission or absorption value. This was the first, and, until the 1950 s, the only approach to scanning cytometry, and, even when measurements were made at the whole cell level, the process was extremely timeconsuming, especially since there was no practical way to store data other than by writing down measured values as one went along. Publications were unlikely to contain data from more than a few hundred cells. By the 1960 s, Zeiss had commercialized a current version of Caspersson's apparatus, and others had begun to build high-resolution scanning microscopes incorporating a variety of technologies. During the 1950s, what we now call "cytometry" was known as "analytical cytology". The first and second editions of a book with the latter title appeared in 1955 and 1959 (Mellors 1959). The book included chapters on histochemistry, on absorption measurement, on phase, interference, and polarizing microscopy, and on Coons's fluorescent antibody method (Coons et al. 1941).

\subsection{2}

\section{Fluorescence Microscopy and the Fluorescent Antibody Technique}

Fluorescence microscopy was developed around the turn of the 20th century. The earliest instruments used UV light for excitation; later systems could employ excitation at blue and longer wavelengths, but the requirement for relatively high power at relatively short wavelengths made it necessary to use arc lamps, rather than filament lamps, as light sources. Fluorescence microscopy, in principle, allows visualization of bright objects against a dark background. Earlier systems, however, were likely to fall short of achieving this goal because they were essen- 
tially transmitted-light microscopes with colored glass filters in both the excitation path, that is, between the light source and the condenser, and the emission path, that is, between the objective and the eyepiece. The combination of stray light transmission through both excitation and emission filters and fluorescence excited in the emission filter often resulted in the background being too bright to permit observation of weakly fluorescent material.

An extremely important application of fluorescence microscopy developed during the 1940s was the fluorescent antibody technique introduced by Coons et al. (1941). Other workers had demonstrated that azo dye-conjugated antisera to bacteria retained their reactivity with the organisms and would agglutinate them to form faintly colored precipitates; however, the absorption of the dye-conjugated sera was not strong enough to permit visual detection of bacterial antigens in tissue preparations.

Albert Coons surmised that it might be easier to detect small concentrations of antibody labeled with fluorescent material against a dark background using fluorescence microscopy. He and his coworkers labelled anti-pneumococcal antibodies with anthracene and could detect both isolated organisms and, more importantly, antibody bound to antigen in tissue specimens, by the UV-excited blue fluorescence of this label, as long as tissue autofluorescence and background were not excessive.

In 1950, Coons and Kaplan reported that fluorescein gave better results as an antibody label than did anthracene, because the blue-excited yellow-green fluorescence of fluorescein was easier to discriminate from autofluorescence. Thereafter, fluorescein became and has remained the most widely used immunofluorescent label.

A significant advance in fluorescence microscopy, epiillumination, was made in 1967 by Ploem (1967), who substituted dichroic mirrors for the half-silvered mirror normally used in an incident light microscope, and added excitation and emission filters to the optical path. Even when color glass filters were still used for excitation and emission wavelength selection, this configuration greatly reduced both stray light transmission and filter fluorescence, yielding much lower backgrounds. Within a short time, it had been reported that, when an epiilluminated apparatus was employed, measurements of nuclei stained by a fluorescent Feulgen procedure using acriflavine yielded results equivalent to those obtained by the standard absorption method (Böhm and Sprenger 1968).

\section{2 .3}

\section{Computers Meet Cytometers: The Birth of Analytical Flow Cytometry}

By the mid-1950s, it had become clear that malignant cells often contained more nucleic acid than normal cells, and Mellors and Silver (1951) proposed construction of an automatic scanning instrument for screening cervical cytology (Papanicolaou or "Pap" smears). Their prototype measured fluorescence rather than absorption, and anticipated Ploem (1967) in introducing UV epillumination. Tolles (1955) described the "Cytoanalyzer" built for cervical cytology. A disc containing a 
series of apertures rotated in the image plane of a transmitted light microscope, producing a raster scan of a specimen with approximately $5-\mu \mathrm{m}$ resolution. A hardwired analyzer extracted nuclear size and density information; cells were then classified as normal or malignant using these parameters. The Cytoanalyzer proved unsuitable for clinical use, but its performance was encouraging enough for the American Cancer Society and the US National Cancer Institute to continue funding research on cytology automation in the United States.

Recording and storing cell images was a nontrivial task in the 1960s, when mainframe computers occupied entire rooms, required kilowatts of power for both the computer and the mandatory air-conditioning, and cost millions of dollars, for which the buyer received a computer with speed and storage capacity exceeded a thousand-fold by a 2005 model laptop costing under US $\$ 1000$. Nonetheless, when minicomputers became available in the middle of the decade, there were at least a few groups of analytical cytologists ready to use them. The TICAS system, assembled at the University of Chicago in the late 1960s, interfaced Zeiss's (Oberkochen, Germany) commercial version of the Caspersson microspectrophotometer to a minicomputer, with the aim of automating interpretation of Pap smears (Wied and Bahr 1970).

The use of stage motion for scanning made operation extremely slow; it could take many minutes to produce a high-resolution scanned image of a single cell, even when there were computers available to capture the data. Somewhat higher speed could be achieved by using discs or galvanometer-driven moving mirrors for image scanning, and limiting the tasks of the motorized stage to bringing a new field of the specimen into view and into focus; this required some electronic storage capability, and made measurements susceptible to errors due to uneven illumination across the field, although this could be compensated for. My colleagues and I at the US National Institutes of Health (NIH; Bethesda, MD, USA) built "Spectre II" (Stein et al. 1969), which incorporated a galvanometer mirror scanning system (Ingram and Preston 1970) and a Digital Equipment Corporation LINC-8 minicomputer. While this system had sufficient computer power to capture high-resolution cell images $(0.2 \mu \mathrm{m}$ pixels $)$, data had to be recorded on 9-track tape and transported to a mainframe elsewhere on the NIH campus for analysis (Shapiro et al. 1971).

Although imaging cytometers of the 1960s were not based on video cameras, for a number of reasons, not least of which was the variable light sensitivity of different regions of a camera tube, which made quantitative measurements difficult, it was recognized that the raster scan mechanism of a cathode ray tube could be used on the illumination side of an image analysis system, with the "flying spot" illuminating only a small segment of the specimen plane at any given time (Young 1951). The CYDAC system, a flying spot scanner built at Airborne Instruments Laboratory (Long Island, NY, USA), was used in studies of the automation of differential leukocyte counting (Prewitt and Mendelsohn 1966) and chromosome analysis (Mendelsohn 1976).

During World War II, the US Army became interested in developing devices for rapid detection of bacterial biowarfare agents in aerosols; this would require pro- 
cessing a relatively large volume of sample in substantially less time than would have been possible using even a low-resolution scanning system. The apparatus built by Gucker et al. (1947) in support of this project achieved the necessary rapid specimen transport by injecting the air stream containing the sample into the center of a larger sheath stream of flowing air that passed through the focal point of a dark-field microscope. Particles passing through the system scattered light into a collection lens, eventually producing electrical signals from a photodetector. The instrument could detect objects in the order of $0.5 \mu \mathrm{m}$ in diameter, and is generally recognized as having been the first flow cytometer used for observation of biological cells. Although Moldavan had suggested counting cells in a fluid stream a decade earlier (Moldavan 1934), his account suggests that he failed to build a working apparatus.

By the late 1940s and early 1950s, the principles of the Gucker apparatus, including the use of sheath flow, were applied to the detection and counting of red blood cells in saline solutions (Crosland-Taylor 1953), providing effective automation for a diagnostic test notorious for its imprecision when performed by a human observer using a hemocytometer and a microscope. Neither the bacterial counter nor the early red cell counters had any significant capacity either for discriminating different types of cells or for making quantitative measurements. Both types of instrument were measuring what users of flow cytometers now call side-scatter signals; although larger particles, in general, produced larger signals than smaller ones, correlations between particle sizes and signal amplitudes were not particularly strong.

An alternative flow-based method for cell counting was developed in the 1950s by Wallace Coulter (Coulter 1956). Recognizing that cells, which are surrounded by a lipid membrane, are relatively poor conductors of electricity as compared to saline, he devised an apparatus in which cells passed one by one through a small $(<100 \mu \mathrm{m})$ orifice between two chambers filled with saline. When a cell passed through, the electrical impedance of the orifice increased in proportion to the volume of the cell, producing a voltage pulse. The Coulter counter (Coulter Electronics, now Beckman Coulter, Hialeah, FL, USA) was widely adopted in clinical laboratories for blood cell counting; it was soon established that it could provide more accurate measurements of cell size than had previously been available (Brecher et al. 1956; Mattern et al. 1957).

In the early 1960s, investigators working with Leitz (Wetzlar, Germany) conceived a hematology counter that added a fluorescence measurement to the light scattering measurement used in red cell counting (Hallermann et al. 1964). If a fluorescent dye such as acridine orange was added to the blood sample, white cells would be stained much more brightly than red cells; the white cell count could then be derived from the fluorescence signal, and the red cell count from the scatter signal. It was also noted that acridine orange fluorescence could be used to discriminate mononuclear cells from granulocytes. It is not clear whether the device, which would have represented a new level of sophistication in flow cytometry, was actually built. 
Around the same time, the promising results obtained with the Cytoanalyzer in attempts to automate reading of Pap smears (Tolles 1955) encouraged executives at the International Business Machines Corporation (IBM, Armonk, NY, USA) to look into producing an improved instrument. Assuming this would be some kind of image analyzer, IBM gave technical responsibility for the program to Louis Kamentsky, who had developed a successful optical character reader. He did some calculations of what would be required in the way of light sources, scanning rates, and computer storage and processing speeds to solve the problem using image analysis, and concluded that a different approach would be required.

Having learned from pathologists in New York that cell size and nucleic acid content could provide a good indicator of whether cervical cells were normal or abnormal, Kamentsky traveled to Caspersson's laboratory in Stockholm and learned microspectrophotometry. He then built a microscope-based flow cytometer that used a transmission measurement at visible wavelengths to estimate cell size and a 260-nm UV absorption measurement to estimate nucleic acid content (Kamentsky 1973; Kamentsky et al. 1965). Subsequent versions of this instrument, which incorporated a dedicated computer system, could measure as many as four cellular parameters (Kamentsky and Melamed 1969). A brief trial on cervical cytology specimens indicated that the system had some ability to discriminate normal from abnormal cells (Koenig et al. 1968); it could also produce distinguishable signals from different types of cells in blood samples stained with a combination of acidic and basic dyes.

The first commercial flow cytometric differential leukocyte counter, introduced in the early 1970s, was the Hemalog D (Technicon Corporation, now Bayer, Tarrytown, NY, USA); Ornstein was a prime mover in its development, having interacted with Kamentsky's group along the way (Ornstein 1987). The Hemalog D analyzed three separate aliquots of sample, making light scattering and absorption measurements at different wavelengths in three different flow cytometers to classify leukocytes based on the relatively specific cytochemical staining procedures used by hematologists for such purposes as determination of lineage of leukemic cells. Although the apparatus performed well, it was initially regarded with a great deal of suspicion, at least in part due to the novelty of flow cytometry. The developers and manufacturers of then-contemporary image analyzing differential counters, which certainly did not perform much better than did the Hemalog D, did what they could to keep potential users suspicious of flow cytometry for as long as possible; the technology would eventually be legitimized by its dramatic impact on immunology, which was facilitated by the introduction of cell sorting and immunofluorescence measurements.

\subsection{4}

\section{The Development of Cell Sorting}

Although impedance (Coulter) counters and optical flow cytometers could analyze hundreds of cells/second, providing a high enough data acquisition rate to be 
useful for clinical use, microscope-based static cytometers offered a significant advantage. A system with computer-controlled stage motion could be programmed to reposition a cell on a slide within the field of view of the objective (Stein et al. 1969), allowing the cell to be identified or otherwise characterized by visual observation; it was, initially, not possible to extract cells with known measured characteristics from a flow cytometer. Until this could be done, it would be difficult to verify any cell classification arrived at using a flow cytometer, especially where the diagnosis of cervical cancer or leukemia might be involved.

This problem was solved in the mid-1960s, when both Mack Fulwyler (Fulwyler 1965), working at the Los Alamos National Laboratory (Los Alamos, NM, USA), and Kamentsky, at IBM (Kamentsky and Melamed 1967), demonstrated cell sorters built as adjuncts to their flow cytometers. Kamentsky's system used a syringe pump to extract selected cells from its relatively slow-flowing sample stream. Fulwyler's was based on ink jet printer technology then recently developed by Richard Sweet (Sweet 1965) at Stanford University (Stanford, CA, USA); following passage through the cytometer's measurement system (originally a Coulter orifice), the saline sample stream was broken into droplets, and those droplets that contained cells with selected measurement values were electrically charged at the droplet break-off point. The selected charged droplets were then deflected into a collection vessel by an electric field; uncharged droplets went, as it were, down the drain.

\section{3}

\section{The Growth of Multiparameter Flow Cytometry}

In the early 1970s, the group at Los Alamos led the way in implementation of practical multiparameter flow cytometers; their larger instruments, with droplet sorting capability, combined two-color fluorescence measurements with measurements of Coulter volume and (thanks to the contributions of Paul Mullaney, Gary Salzman, and others) light scattering at several angles (Mullaney et al. 1969; Salzman et al. 1975a, 1975b; Steinkamp et al. 1973). The cytometers were interfaced to Digital Equipment Corporation (Maynard, MA, USA) minicomputers. Several instruments made at Los Alamos were delivered to the National Institutes of Health; other institutions copied most or all of the Los Alamos design in their own laboratory-built apparatus.

Fluorescence measurement had been introduced to flow cytometry in the late 1960s to improve both quantitative and qualitative analyses. By that time, Van Dilla et al. (1969) at Los Alamos and Dittrich and Göhde (1969) in Germany had built fluorescence flow cytometers to measure cellular DNA content; the Los Alamos investigators used a fluorescent Feulgen staining procedure, whereas Dittrich and Göhde's publication introduced the use of ethidium bromide as a rapid DNA stain, facilitating analysis of abnormalities in tumor cells and of cell cycle kinetics in both neoplastic and normal cells. The Los Alamos instrument incorporated the orthogonal "body plan" now standard in laser-source instruments, with 

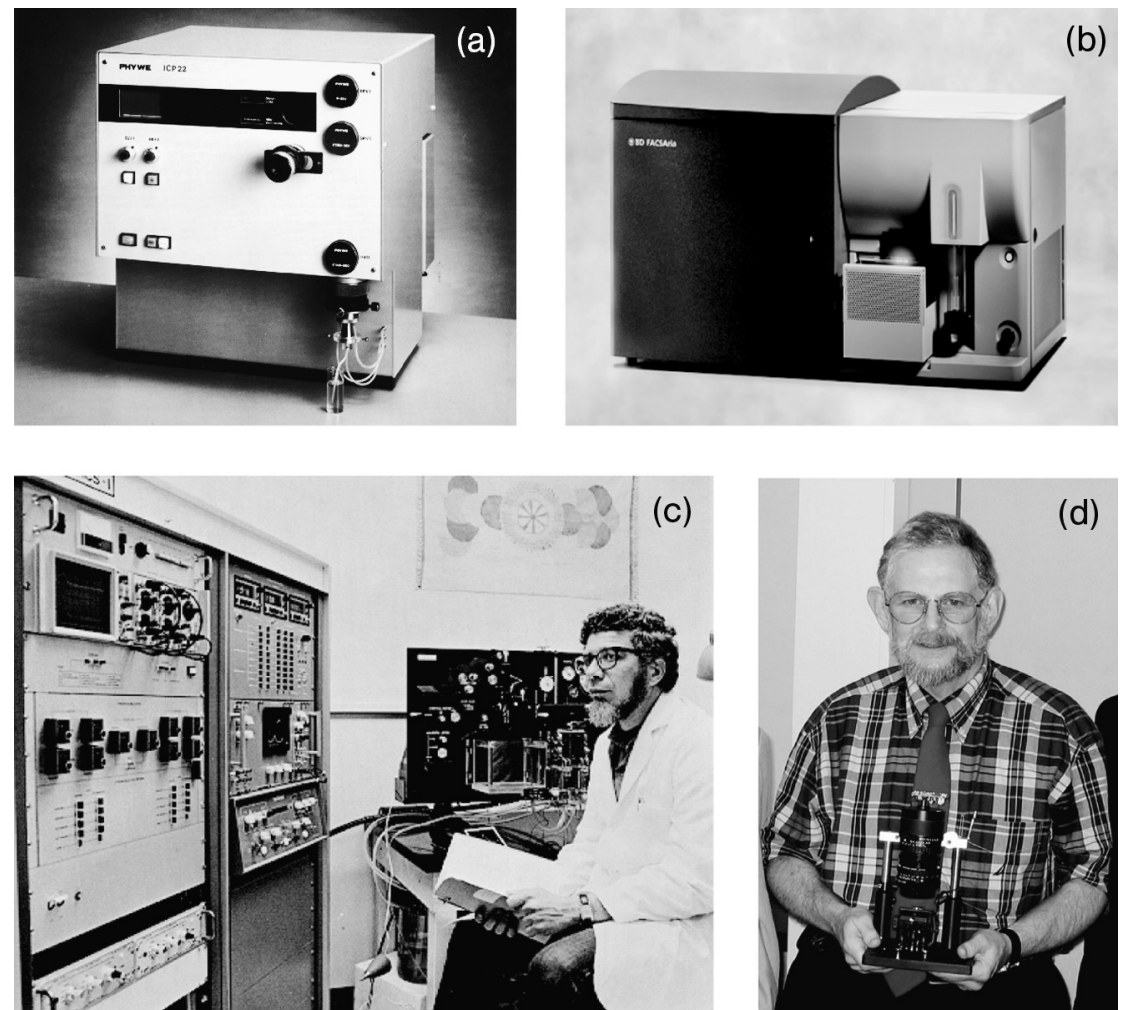

Fig. 1.1 Three historic flow cytometers and a post-historic image cytometer. (a) Phywe Impulscytophotometer ICP 22, which was licensed to Phywe by Partec, was one of the first compact bench-top flow cytometers (1975). (b) The BD Biosciences FACSAria, a modern high-speed cell sorter reduced to the size of a typical bench-top instrument (BD Biosciences). (c) A $1974 \mathrm{NIH}$ photograph of Leonard Herzenberg with the B-D FACS-1, the first commercial cell sorter. (d) The author with a simple imaging cytometer, minus the laptop computer used for data collection and analysis.

the optical axes of illumination and light collection at right angles to each other and to the direction of sample flow. Kamentsky, who had left IBM to found Bio/ Physics Systems (Mahopac, NY, USA), produced the Cytofluorograf, an orthogonal geometry fluorescence flow cytometer that was the first commercial product to incorporate an argon ion laser; Wolfgang Göhde's Partec (Münster, Germany) Impulscytophotometer (ICP) instrument built around a fluorescence microscope with arc lamp illumination, was distributed commercially by Phywe (Göttingen, Germany). Using this instrument, Heller (1973) was the first to describe flow cytometric measurements of DNA content in plant cell nuclei (see Chapter 3). Partec instruments (Fig. 1.1a), which are relatively compact and, in their simpler configurations, can be run on batteries, remain popular for use in botanical applications. 
Leonard Herzenberg and his colleagues (Herzenberg et al. 1976) at Stanford, realizing that fluorescence flow cytometry and subsequent cell sorting could provide a useful and novel method for purifying living cells for further study, developed a series of instruments after exposure to a Kamentsky prototype lent by IBM (Saunders and Hulett 1969). Although their original apparatus (Hulett et al. 1969), with arc lamp illumination, was not sufficiently sensitive to permit them to achieve their objective of sorting cells from the immune system, based on the presence and intensity of staining by fluorescently labelled antibodies, the second version (Bonner et al. 1972), which used a water-cooled argon laser, was more than adequate. This was commercialized as the Fluorescence-Activated Cell Sorter (FACS) in 1974 by Becton-Dickinson (B-D, now BD Biosciences, San Jose, CA, USA) (Fig. 1.1b).

Coulter Electronics (now Beckman Coulter), which by 1970 had become a very large and successful manufacturer of laboratory hematology counters, pursued the development of fluorescence flow cytometers through a subsidiary, Particle Technology, under Mack Fulwyler's direction in Los Alamos. The TPS-1 (Two Parameter Sorter), Coulter's first product in this area, reached the market in 1975. It used an air-cooled 35-mW argon ion laser source and could measure forward scatter and fluorescence.

Multiple wavelength fluorescence excitation was introduced to flow cytometry in apparatus built at Block Engineering (Cambridge, MA, USA) during an abortive attempt to develop a hematology instrument. The first instrument (Curbelo et al. 1976) derived five illuminating beams from a single arc lamp; the second (Shapiro et al. 1977) used three laser beams; both could analyze over 30000 cells per second and, using hardwired pre-processors and integral minicomputers, identify cells comprising less than 1/100 000 of the total sample. The laser source system incorporated forward and side scatter measurements, which permitted lymphocyte gating (Shapiro 1977), influenced by work carried out at Los Alamos (Salzman et al. 1975a, 1975b). Block also built a slow flow system intended for detection of hepatitis B virus and antigen in serum; it could discriminate scatter singles from large viruses (Hercher et al. 1979) and could theoretically detect a few dozen fluorescein molecules above background. The Block cytometers were never sold commercially, but influenced the optical, electronic, and systems design of later instruments.

By the time the Society for Analytical Cytology (now ISAC) came into being in 1978, B-D, Coulter, and Ortho (a division of Johnson \& Johnson that bought Bio/ Physics Systems and was ultimately acquired by B-D) were producing flow cytometers that could measure small- (forward scatter) and large- (side scatter) angle light scattering and fluorescence in at least two wavelength regions, analyzing several thousand cells per second, and with droplet deflection cell sorting capability. Ortho was also distributing the ICP, which, by virtue of its optical design, could make higher precision measurements of DNA content than could laserbased flow cytometers. DNA content analysis was receiving considerable attention as a means of characterizing the aggressiveness of breast cancer and other malignancies, and, at least in part due to the results of a Herzenberg sabbatical in 
Cesar Milstein's laboratory in Cambridge (UK), monoclonal antibodies had begun to emerge as practical reagents for dissecting the stages of development of cells of the blood and immune system. Loken, Parks, and Herzenberg had successfully performed a two-color immunofluorescence experiment, introducing fluorescence compensation in the process (Loken et al. 1977), although it was clear that a great deal needed to be done in the area of fluorescent-label development to realize the potential of monoclonal antibodies.

Although the instruments Kamentsky built at IBM were computer controlled, computers were expensive options for most flow cytometers until the early 1980s, by which time microprocessor-based systems could do the work of an earlier generation of minicomputers. Without computers, instruments might be able to measure four or more parameters per cell, but did not have the processing power to implement true multiparameter methodology for gating, that is, selection of subsets of cells from a heterogeneous population using combinations of several measurement values, or for sort control. Once dedicated microcomputers became "standard equipment" on commercial flow cytometers, multiparameter measurement techniques became practical for many more researchers than had previously been able to use them.

\section{4}

\section{Bench-tops and Behemoths: Convergent Evolution}

From the early 1970 s on, commercial production of instruments has allowed researchers who cannot develop and build their own apparatus to pursue increasingly sophisticated applications of fluorescence flow cytometry and sorting. Advances in the technology itself have continued to occur primarily in a relatively small community of academic, government, and industrial laboratories.

Los Alamos provided the inoculum for the subsequent growth of another major center for flow cytometer development at Lawrence Livermore Laboratory (Livermore, CA, USA), where high-speed flow sorting was perfected as a means for separating human chromosomes stained with a combination of A-T selective (Hoechst 33342) and G-C selective (chromomycin $A_{3}$ ) DNA dyes (Gray et al. 1987; Peters et al. 1985). The MoFlo high-speed sorter developed by Ger van den Engh and others at Livermore was subsequently refined by Cytomation (now DakoCytomation, Fort Collins, CO, USA), and has been produced commercially by them since 1994 .

Chromosome sorting (also of major interest to botanists (Doležel et al. 2001; Chapter 16)) and high-speed sorting in general initially required high-powered, water-cooled ion lasers, in part because the first generation of high-speed instruments from B-D and Cytomation made measurements of cells in a jet in air, necessitating the use of relatively inefficient light collection optics. Systems built around fluorescence microscopes, such as the original Partec Impulscytophotometer and the system originally described by Tore Lindmo and Harald Steen in 1979 (Steen 1980; Steen and Lindmo 1979), can make optimal use of the relatively low 
excitation power available from mercury or xenon arc lamps by using high numerical aperture microscope lenses or their equivalent for both illumination and light collection. The Lindmo-Steen apparatus, sensitive enough to make precise DNA content measurements of bacteria (Steen et al. 1982), was originally commercialized by Leitz; later versions were sold by Skatron (Lier, Norway) and BioRad (Milano, Italy), and the latest, now being produced by Apogee (Hemel Hempstead, Herts, UK) can use a low-power laser source to measure scatter signals from viruses (Steen 2004). Another bench-top arc source flow cytometer, the Quanta, developed by NPE Systems (Miami, FL, USA), makes both fluorescence and impedance measurements, and is now being sold by Beckman Coulter.

In the mid and late 1970s, Kamentsky's Bio/Physics Systems and its successor, Ortho Diagnostics Systems, introduced laser source flow cytometers and sorters in which measurements were made in flat-sided quartz flow cuvettes, and in which "high-dry" microscope objectives were used to increase light collection. This made it possible to use air-cooled rather than water-cooled lasers for immunofluorescence measurements, decreasing the size, cost, and power consumption of instruments. In the early 1980s, B-D introduced its FACS analyzer, a small but sensitive bench-top system employing an arc lamp source; within a few years, it was supplanted by the FACScan, a three-color bench-top analyzer using a rectangular cuvette with a gel-coupled lens for highly efficient light collection, allowing more sensitive immunofluorescence measurements to be made using a $15-\mathrm{mW}$ air-cooled argon laser source than were possible using 10 times more laser power in jet-in-air sorters. The FACScan was followed by the FACSort, which included a relatively slow fluidic sorter; both were succeeded by the FACSCalibur, which offers both a fluidic sorting option and a fourth fluorescence channel with excitation from a red (635-640 $\mathrm{nm}$ ) diode laser.

The emphasis in the Herzenbergs' laboratory at Stanford has remained on sorting cells on the basis of immunofluorescence signals with the aim of isolating morphologically indistinguishable viable lymphocytes with differences in functional characteristics. This required development of a large armamentarium of monoclonal antibodies, of labels with diverse spectral characteristics, and of the hardware and software necessary to achieve multiparameter fluorescence compensation (Bagwell and Adams 1993) and gating rapidly enough to implement complex sorting strategies. Until the algal photosynthetic pigment phycoerythrin was introduced as an antibody label in 1982 (Oi et al. 1982), two large lasers were required for two-colour immunofluorescence measurements; the combination of fluorescein- and phycoerythrin-labeled antibodies could be excited effectively at $488 \mathrm{~nm}$, while providing sufficient separation of emission maxima to discriminate the fluorescence of the two labels. It is now possible, using a combination of organic dye labels, phycobiliproteins, tandem conjugates of both, and semiconductor nanocrystals ("quantum dots") as labels, to carry out 17-color immunofluorescence experiments on flow cytometers with three laser beams (Perfetto et al. 2004). Large lasers are no longer required.

From the mid-1990s on, there has been a proliferation of diode and solid-state lasers, and these small, energy-efficient, and (usually) relatively inexpensive 
sources have increasingly been incorporated into flow cytometers. The use of violet $(395-415 \mathrm{~nm})$ diode lasers in cytometry was first described at a meeting in 2000 (Shapiro and Perlmutter 2001); by 2002, several manufacturers had incorporated such sources into their instruments. They can be used to excite DNA dyes such as DAPI and Hoechst 33342, which are normally used with UV excitation. Frequency-doubled diode-pumped YAG lasers, emitting green light at $532 \mathrm{~nm}$, have also come into use, and are available in bench-top analyzers from BD Biosciences, Guava Technologies (Hayward, CA, USA), and Luminex (Austin, TX); all but the last of these companies also offer doubled semiconductor lasers emitting at $488-492 \mathrm{~nm}$ in lieu of argon lasers.

An increasing amount of the internal electronics of flow cytometers has become computer-based, with the latest systems incorporating special-purpose large-scale integrated circuits, microprocessors, microcontrollers, and digital signal-processing chips.

The development of digital audio, telephony, and video has resulted in large increases in the performance, and decreases in the price, of analog-to-digital converters (ADCs), which are critical elements in data acquisition systems for any type of instrumentation, flow cytometers included. The ADCs originally used with flow cytometers had only 8- or 10-bit resolution, making it necessary to use logarithmic amplifiers to process signals with a large dynamic range. This necessitated the use of hardware for fluorescence compensation. While this approach is feasible when three or four colors are measured, it is essentially impossible to implement for modern multibeam instruments in which measurements of 12 or more colors may be made. The alternative is software compensation (Bagwell and Adams 1993), which is best applied to linear data digitized to at least 16-bit resolution. In the early 1990 s, software compensation was implemented in the Beckman Coulter EPICS XL analyzer, which captures 20-bit linear data, eliminating the need for logarithmic amplifiers. BD Biosciences's DiVa electronics use highspeed digitization to permit digital computation of pulse height, width, and area, while DakoCytomation and Partec have developed their own approaches to highresolution digital data analysis. As has been the case for audio and video, digital techniques can be expected to become predominant in cytometry.

With the introduction of the FACSAria sorter (Fig. 1.1c) in late 2002, BD Biosciences successfully hybridized the behemoth high-speed cell sorter and the bench-top analyzer; this bench-top apparatus incorporates digital electronics and measures cells in a cuvette using as many as three beams, typically at 407, 488, and $633 \mathrm{~nm}$, all derived from low-power, air-cooled lasers. The InFlux, a highspeed sorter recently introduced by Ger van den Engh's new company, Cytopeia (Seattle, WA, USA), also features a small footprint and the ability to operate using only air-cooled lasers.

Multilaser bench-top analyzers are now available from BD Biosciences, Beckman Coulter, DakoCytomation, Luminex, and Partec; the latter also offers a combination of arc lamp and laser sources. Users not completely satisfied with the multiparameter software available from their cytometer manufacturers can choose from among the offerings of a number of third-party providers. 
With the aid of a continually expanding repertoire of reagents, many of which are exquisitely specific, an ever more colorful palette of fluorescent labels, and increasingly sophisticated data analysis procedures, multiparameter fluorescence flow cytometers can identify many more lineages and sublineages of both prokaryotic and eukaryotic cells than were contemplated a few decades ago. Remarkably, all of the work is carried out without the benefit of morphologic information. Although the instruments also typically measure small-angle or forward light scatter, often erroneously referred to as a "size" measurement, and largeangle or side scatter, which provides information about internal granularity and surface roughness but does not resolve cellular detail, most of the information needed for cell identification comes from the intensity of fluorescence measurements made at various excitation and emission wavelengths.

Flow cytometry remains a highly effective, accurate, and precise way to obtain objective and quantitative information from single cells; its principal disadvantages lie in the complexity and cost of the apparatus. It is thus logical to ask whether the advances made in electronics and electro-optics in recent years might provide a simpler and more affordable alternative. Recent work in my laboratory (Shapiro 2004b; Shapiro and Perlmutter 2006) and elsewhere (Jelinek et al. 2001; Mazzini et al. 2005; Rodriquez et al. 2005; Stothard et al. 2005; Varga et al. 2004; Wittrup et al. 1994) provides grounds for optimism.

\section{5}

\section{Image Cytometry: New Beginnings?}

In 1994, Wittrup et al. described a cytometric apparatus called the Fluorescence Array Detector (FAD), in which camera lenses were used to form a 1:1 image of a $1 \times 1 \mathrm{~cm}$ field of view on a cooled $512 \times 512$ pixel charge-coupled device (CCD) detector with $20 \mu \mathrm{m}$ square pixels. Since each pixel collected light from an area larger than the area of a typical cell, no morphologic information was available. The instrument had only one moving part, a focusing stage; low-intensity $\left(1 \mathrm{~mW} / \mathrm{cm}^{2}\right)$ illumination of the field came from the expanded beam of a 488$\mathrm{nm}$ air-cooled argon ion laser. Although a software shading correction was used in an attempt to compensate for the uneven illumination obtained from the laser beam, the coefficient of variation $(\mathrm{CV})$ of the fluorescence intensity distribution of 6- $\mu \mathrm{m}$ polystyrene beads was reported to be $12.9 \%$; this relatively large variance, which would be unacceptable in the context of DNA content measurement, was attributed to imperfect shading correction. Sensitivity was impressive; noise due to dark current and stray light was equivalent to only a few hundred fluorescein MESF (molecules of equivalent soluble fluorochrome)/pixel, although fluorescence from conventional glass microscope slides increased the background fluorescence by approximately 10 -fold.

At the time the FAD was constructed, a cooled CCD camera cost tens of thousands of dollars; and a 50-mW air-cooled 488-nm argon laser cost at least $\$ 8000$, eliminating the instrument from consideration as a low-cost replacement for a 
flow cytometer. At present, CCD and complementary metal oxide-silicon (CMOS) cameras with the requisite sensitivity are available for no more than a few hundred dollars; however, argon ion lasers and solid-state lasers emitting at the same wavelength still cost thousands of dollars. While in 1994, an arc lamp, also costing thousands of dollars, would probably have been the only feasible alternative to a laser as the light source for an instrument similar to the FAD, it has recently been shown (Mazzini et al. 2005) that high-intensity light-emitting diodes (LEDs), available for only a few dollars, can provide sufficient illumination over the required area to be usable as light sources for fluorescence microscopy as well as for an FAD-like device. We have established (Shapiro and Perlmutter 2006) that an apparatus using an LED for excitation, relatively inexpensive 35$\mathrm{mm}$ camera lenses or low power microscope lenses for light collection, and a CCD or CMOS camera as a detector (Fig. 1.1d) can detect low-level fluorescence signals in the range that would be expected from cells stained with fluorescent antibodies, as well as the substantially stronger signals associated with cells stained for DNA. Although we have not definitively established a range of measurement precision, we note that Varga et al. (2004) achieved CVs of less than $4 \%$ by applying a correction for uneven illumination to fluorescence measurements made using a CCD camera on a conventional fluorescence microscope, and believe that at least equivalent performance can be achieved in a large-field imaging system analogous to the FAD.

Rodriguez et al. (2005) have demonstrated that a prototype low-magnification imaging system can be as effective as a flow cytometer for immunofluorescencebased counting of CD4+ T lymphocytes in the blood of patients infected with HIV, and there is evidence that well-designed, extremely inexpensive "toy" microscopes are sufficiently rugged and of sufficient optical quality for use in tropical environments (Jelinek et al. 2001; Stothard et al. 2005). Thus, a new generation of image cytometers, under development to facilitate infectious disease diagnosis in resource-poor countries, may soon provide botanists with an effective and economical means of determining genome sizes of plants in the field, a welcome and not completely unintended consequence.

\section{Acknowledgment}

The author thanks Dr. Roland Göhde (Partec GmbH, Münster, Germany) for the photo of the Phywe Impulscytophotometer ICP 22 in Fig. 1.1a.

\section{References}

Bagwell, C. B., Adams, E. G. 1993, Ann. NY Acad. Sci. 677, 167-184.

Baker, J. R. 1958, Principles of Biological Microtechnique, Methuen, UK.
Bennett, M. D., Leitch, I. J. 2005, Ann. Bot. 95, 1-6.

Böhm, N., Sprenger, E. 1968, Histochemie 16, $100-118$. 
Bonner, W. A., Hulett, H. R., Sweet, R. G., Herzenberg, L. A. 1972, Rev. Sci. Instr. 43 , 404-409.

Brecher, G., Schneiderman, M. G., Williams, Z. 1956, Am. J. Clin. Pathol. 26, 1439-1449.

Breidbach, O. 2002, J. History Biol. 35, 221-250.

Caspersson, T. O. 1950, Cell Growth and Cell Function, Norton, USA.

Caspersson, T. O., Schultz, J. 1938, Nature 142, 294-295.

Clark, G., Kasten, F. H. 1983, History of Staining, 2nd edn, Williams \& Wilkins, USA.

Coons, A. H., Kaplan, M. H. 1950, J. Exp. Med. 91, 1-13.

Coons, A. H., Creech, H. J., Jones, R. N. 1941, Proc. Soc. Exp. Biol. Med. 47, 200-202.

Coulter, W. H. 1956, Proc. Natl Elect. Conf. 12, 1034-1042.

Crosland-Taylor, P. J. 1953, Nature 171, 37-38.

Curbelo, R., Schildkraut, E. R., Hirschfeld,

T., Webb, R. H., Block, M. J., Shapiro, H. M. 1976, J. Histochem. Cytochem. 24, 388-395.

Dittrich, W., Göhde, W. 1969, Zeitschrift Naturforsch. 24b, 360-361.

Doležel, J., Bartoš, J. 2005, Ann. Bot. 95, 99-110.

Doležel, J., Lysák, M. A., Kubaláková, M., Šimková, H., Macas, J., Lucretti, S. 2001, Methods Cell Biol. 64, 3-31.

Feulgen, R., Rossenbeck, H. 1924, HoppeSeyler's zeitschr. physiol. Chemie 135, 203248.

Fulwyler, M. J. 1965, Science 150, 910-911.

Gray, J. W., Dean, P. N., Fuscoe, J. C., Peters, D. C., Trask, B. J., van den Engh, G. J., Van Dilla, M. A. 1987, Science 238, 323-329.

Greilhuber, J., Doležel, J., Lysák, M. A., Bennett, M. D. 2005, Ann. Bot. 95, 255-260.

Gucker Jr., F. T., O’Konski, C. T., Pickard, H. B., Pitts Jr., J. N. 1947, J. Am. Chem. Soc. 69, 2422-2431.

Hallermann, L., Thom, R., Gerhartz, H. 1964, Verhand. Deutsch. Gesellschaft Inn. Med. 70, 217-219.

Heller, F. O. 1973, Ber. Deutsch. Bot. Gesellschaft 86, 437-441.

Hercher, M., Mueller, W., Shapiro, H. M. 1979, J. Histochem. Cytochem. 27, 350-352.

Herzenberg, L. A., Sweet, R. G., Herzenberg, L. A. 1976, Scientific American 234, 108-117.
Hulett, H. R., Bonner, W. A., Barrett, J., Herzenberg, L. A. 1969, Science 166, 747-749.

Ingram, M., Preston Jr., K. 1970, Scientific American 223, 72-78.

Jelinek, L., Peters, G., Okuley, J., McGowan, S. 2001, Intel Technol. J. 5, 1-10.

Kamentsky, L. A. 1973, Adv. Biol. Med. Phys. 14, 93-161.

Kamentsky, L. A., Melamed, M. R. 1967, Science 156, 1364-1365.

Kamentsky, L. A., Melamed, M. R. 1969, Proc. IEEE 57, 2007-2016.

Kamentsky, L. A., Melamed, M. R., Derman, H. 1965, Science 150, 630-631.

Koenig, S. H., Brown, R. D., Kamentsky, L. A., Sedlis, A., Melamed, M. R. 1968, Cancer 21, 1019-1026.

Loken, M. R., Parks, D. R., Herzenberg, L. A. 1977, J. Histochem. Cytochem. 25, 899-907.

Mattern, C. F. T., Brackett, F. S., Olson, B. J. 1957, J. Appl. Physiol. 10, 56-70.

Mazzini, G., Ferrari, C., Baraldo, N., Mazzini, M., Angelini, M. 2005, Improvements in fluorescence microscopy allowed by high power light emitting diodes, in Current Issues on Multidisciplinary Microscopy Research and Education, (vol. 2), ed. A. Méndez-Vilas, L. Labajos-Broncano, Formatex, Spain, pp. 181-188.

Mellors, R. C. (ed.) 1959, Analytical Cytology, 2nd edn, McGraw-Hill, USA.

Mellors, R. C., Silver, R. 1951, Science 114, 356-360.

Mendelsohn, M. L. (ed.) 1976, Automation of Cytogenetics, CONF-751158, Lawrence Livermore Laboratory, USA.

Moldavan, A. 1934, Science 80, 188.

Mullaney, P. F., Van Dilla, M. A., Coulter, J. R., Dean, P. N. 1969, Rev. Scient. Instr. 40, 1029-1032.

Oi, V. T., Glazer, A. N., Stryer, L. 1982, J. Cell Biol. 93, 981-986.

Ornstein, L. 1987, Electrophoresis 8, 3-13.

Perfetto, S. P., Chattopadhyay, P. K., Roederer, M. 2004, Nature Rev. Immunol. 4, 648-655.

Peters, D. C., Branscomb, E., Dean, P. N., Merrill, T., Pinkel, D., Van Dilla, M. A., Gray, J. W. 1985, Cytometry 6, 290-301.

Ploem, J. S. 1967, Zeitschrift Wissenschaft. Mikrosk. 68, 129-142.

Prewitt, J. M. S., Mendelsohn, M. L. 1966, Ann. NY Acad. Sci. 128, 1035-1053. 
Rodriguez, W. R., Christodoulides, N., Floriano, P. N., Graham, S., Mohanty, S., Dixon, M., Hsiang, M., Peter, T., Zavahir, S., Thior, I., Romanovicz, D., Bernard, B., Goodey, A. P., Walker, B. D., McDevitt, J. T. 2005, PLoS Med. 2, 663-672.

Salzman, G. C., Crowell, J. M., Goad, C. A., Hansen, K. M., Hiebert, R. D., LaBauve, P. M., Martin, J. C., Ingram, M., Mullaney, P. F. 1975a, Clin. Chem. 21, 1297-1304.

Salzman, G. C., Crowell, J. M., Martin, J. C., Trujillo, T. T., Romero, A., Mullaney, P. F., LaBauve, P. M. 1975b, Acta Cytol. 19, 374-377.

Saunders, A. M., Hulett, H. R. 1969, J. Histochem. Cytochem. 17, 188.

Shapiro, H. M. 1977, J. Histochem. Cytochem. 25, 976-989.

Shapiro, H. M. 2003, Practical Flow Cytometry, 4th edn, Wiley-Liss, USA.

Shapiro, H. M. 2004a, Cytometry 58A, 13-20.

Shapiro, H. M. 2004b, Cytometry 60A, 115-124.

Shapiro, H. M., Perlmutter, N. G. 2001, Cytometry 44, 133-136.

Shapiro, H. M., Perlmutter, N. G. 2006, Cytometry 69A, 620-630.

Shapiro, H. M., Bryan, S. D., Lipkin, L. E., Stein, P. G., Lemkin, P. F. 1971, Exp. Cell Res. 67, 81-85.

Shapiro, H. M., Schildkraut, E. R., Curbelo, R., Turner, R. B., Webb, R. H., Brown, D. C., Block, M. J. 1977, J. Histochem. Cytochem. 25, 836-844.

Steen, H. B. 1980, Cytometry 1, 26-31.
Steen, H. B. Cytometry 2004, 57A, 94-99.

Steen, H. B., Lindmo, T. 1979, Science 204, 403-404.

Steen, H. B., Boye, E., Skarstad, K., Bloom, B., Godal, T., Mustafa, S. 1982, Cytometry 2, 249-257.

Stein, P. G., Lipkin, L. E., Shapiro, H. M. 1969, Science 166, 328-333.

Steinkamp, J. A., Fulwyler, M. J., Coulter, J. R., Hiebert, R. D., Horney, J. L., Mullaney, P. F. 1973, Rev. Scient. Instr. 44, 1301-1310.

Stothard, J. R., Kabatereine, N. B., Tukahebwa, E. M., Kazibwe, F., Mathieson, W., Webster, J. P., Fenwick, A. 2005, Am. J. Trop. Med. Hyg. 73, 949955.

Sweet, R. G. 1965, Rev. Scient. Instr. 36, 131-136.

Swift, H. 1950, Proc. Natl Acad. Sci., Washington 36, 643-654.

Tolles, W. E. 1955, Trans. NY Acad. Sci. 17, 250-256.

Van Dilla, M. A., Trujillo, T. T., Mullaney, P. F., Coulter, J. R. 1969, Science 163, 1213-1214.

Varga, V. S., Bocsi, J., Sipos, F., Csendes, G., Tulassay, Z., Molnár, B. 2004, Cytometry 60A, 53-62.

Wied, G. L., Bahr, G. F. (eds.) 1970, Automated Cell Identification and Cell Sorting, Academic Press, USA.

Wittrup, K. D., Westerman, R. J., Desai, R. 1994, Cytometry 16, 206-213.

Young, J. Z. 1951, Nature 167, 231. 
